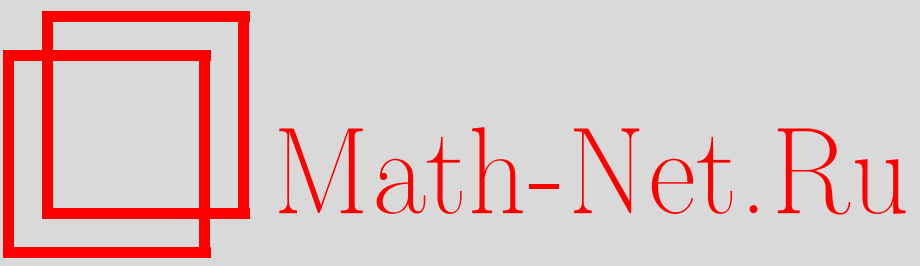

Г. А. Джанашия, Е. Т. Лагвилава, Л. Н. Эпремидзе, О приближенной факторизации положительно определенной матриц-функции, УМН, 1999, том 54, выпуск 6, 161-162

DOI: https://doi.org/10.4213/rm238

Использование Общероссийского математического портала Math-Net.Ru подразумевает, что вы прочитали и согласны с пользовательским соглашением

http://www. mathnet.ru/rus/agreement

Параметры загрузки:

IP: 54.209 .52 .79

26 апреля 2023 г., 07:42:45 


\title{
О ПРИБЛИЖЕННОЙ ФАКТОРИЗАЦИИ ПОЛОЖИТЕЛЬНО ОПРЕДЕЛЕННОЙ МАТРИЦ-ФУНКЦИИ
}

\author{
Г. А. ДЖАНАШИЯ, Е. Т. ЛАГВИЛАВА, Л.Н. ЭПРЕМИДЗЕ
}

В [1], [2] Винер показал, что для положительно определенной матриц-функции

$$
S(t)=\left(f_{i j}(t)\right)_{i, j=\overline{1, r}},
$$

$|t|=1$, с интегрируемыми членами, $f_{i j}(t) \in L_{1}$, если логарифмм детерминанта интегрируем,

$$
\log \operatorname{det}(S(t)) \in L_{1},
$$

то существует факторизация

$$
S(t)=\chi^{+}(t) \cdot\left(\chi^{+}(t)\right)^{*}
$$

где $\chi^{+}$- внешняя аналитическая матриц-функция с членами из класса Харди $H_{2}$, a $\left(\chi^{+}\right)^{*}$ - ее эрмитово сопряжение (в (3) подразумевается, что $\chi^{+}(t)=\left.\chi^{+}(z)\right|_{|z|=t}$ и равенство выполняются почти всюду).

В целях единственности факторизации (3) (она единственна с точностью до постоянного унитарного множителя с правой стороны) будем требовать, чтобы матрица $\chi^{+}(0)$ была положительно определена.

Условие (2) является также необходимым для существования такой факторизации.

Создавая теорию линейного прогнозирования стационарных процессов, Колмогоров и Винер показали, что если (1) - спектральная матриц-функция такого процесса, то его коэффициенты Вольда совпадают с коэффициентами аналитической матриц-функции $\chi^{+}(z)=\sum_{k=0}^{\infty} C_{k} z^{k}$ (см. [3], [2]). По этим коэффициентам, в свою очередь, легко вычисляются коэффициенты прогноза. Таким образом, Колмогоровым и Винером нахождение прогноза для стационарного процесса было сведено к решению задачи факторизации положительно определенной матриц-функции. В общем случае факторизацию (3) в явном виде решить невозможно. Поэтому существенный интерес приобретают методы приближенного нахождения $\chi^{+}$(см. [4]-[6]).

Очень часто для данного стационарного процесса строится эмпирическая спектральная матриц-функция, которая является лишь приближением теоретически существующей спектральной матриц-функции. Так как, вообще говоря, факторизация не устойчива, т.е. "малое" изменение матриц-функции $S(t)$ может привести к “болшшому" изменению матриц-функции $\chi^{+}(t)$, естественно ставится вопрос: насколько мы можем быть уверены в том, что коэффициенты Вольда, соответствующие эмпирической спектральной матриц-функции, остаются близки к коэффициентам Вольда данного стационарного процесса.

На фоне сказанного выше для приложений может оказаться интересной следующая

Tеорема. Пусть $S_{n}(t), n=1,2, \ldots, u S(t)$ - положительно определеннье матриц-функции с интегрируемым логарифмом детерминантов, и имеет место сходимость

$$
\left\|S_{n}(t)-S(t)\right\|_{L_{1}} \rightarrow 0
$$

(подразумевается почленная сходимость в $L_{1}$ ). В этом случае

$$
\left\|\chi_{n}^{+}(t)-\chi^{+}(t)\right\|_{H_{2}} \rightarrow 0
$$

справедливо тогда и только тогда, когда

$$
\log \operatorname{det}\left(S_{n}(t)\right) \stackrel{\text { слабо }}{\longrightarrow} \log \operatorname{det}(S(t))
$$


( под слабой сходимостью последовательности из $L_{1}$ подразумеваем слабую сходимость последовательности соответствующих функционалов на классе непрерывных функций $C[0,2 \pi))$.

Так как $\operatorname{det}\left(\chi_{n}^{+}(z)\right)$ и $\operatorname{det}\left(\chi^{+}(z)\right)$ внешние аналитические функции из класса Неванлинны, из (5) легко следует, что

$$
\log \left|\operatorname{det}\left(\chi_{n}^{+}(t)\right)\right| \stackrel{\text { слабо }}{\longrightarrow} \log \left|\operatorname{det}\left(\chi^{+}(t)\right)\right|,
$$

что означает, ввиду (3), справедливость (6).

Доказательство достаточной части теоремы существенно опирается на алгориффм факторизации положительно определенной матриц-функции, предложенный в [6].

В этой статье для $S(t)$ строится последовательность $\left(S_{n}(t)\right)_{n=1}^{\infty}$ с тем же детерминантом, $\operatorname{det}(S(t))=\operatorname{det}\left(S_{n}(t)\right)$, удовлетворяющая $(4)$, для которой $\chi_{n}^{+}(t)$ найдено в явном виде и выполняется (5).

В общем случае сформулированная теорема не дает ответа на практически важный вопрос: каков порядок сходимости в (5)?

\section{СПИСОК ЛИТЕРАТУРЫ}

[1] Wiener N., Masani P. // Acta Math. 1957. V. 98. P. 111-150. [2] Wiener N., Akutowicz E. J. // J. Math. Mech. 1959. V. 8. P. 111-120. [3] Колмогоров А. Н. // Бюлл. МГУ. 1941. T. 2. №6. C. 1-40. [4] Wiener N., Masani P. // Acta Math. 1958. V. 99. P. 93-137. [5] Masani P. // Acta Math. 1960. V. 104. P. 141-162. [6] Janashia G., Lagvilava E. // Studia Math. 1999. V. 137. № 1. P. 93-100.

Тбилисский математический институт им. Размадзе АН Грузии

Принято редколлегией

02.10.1999 